\title{
An Approximation Algorithm for the Risk-Adjusted Two-Stage Stochastic Facility Location Problem with Penalties
}

\author{
Jiating Shao • Dachuan Xu
}

Received: 4 June 2013 / Revised: 11 July 2013 / Accepted: 17 July 2013 /

Published online: 30 August 2013

(C) Operations Research Society of China, Periodicals Agency of Shanghai University, and

Springer-Verlag Berlin Heidelberg 2013

\begin{abstract}
In this paper, we consider the risk-adjusted two-stage stochastic facility location problem with penalties (RSFLPP). Using the monotonicity and positive homogeneity of the risk measure function, we present an LP-rounding-based 6-approximation algorithm.
\end{abstract}

Keywords Facility location · Approximation algorithm · LP-rounding · Risk-adjusted

Mathematics Subject Classification (2010) 90C27 · 68W25

\section{Introduction}

In the classical facility location problem (FLP), there is a metric space which is made up of the nodes of facilities and clients. We want to open some facilities and assign each client to an open facility. The objective is to minimize the total cost which including the facility opening cost and assignment cost. In the Operations Research community, many researchers devote to design the approximation algorithms for the FLP $[1,4,6,8,9,11]$. The currently best known approximation ratio is 1.488 which is due to $\mathrm{Li}$ [10]. The LP-rounding technique is one of the important techniques for designing the approximation algorithms $[2,13,15]$.

This work was supported by Scientific Research Common Program of Beijing Municipal Commission of Education (No. KM201210005033) and China Scholarship Council.

J. Shao · D. Xu (凶)

Department of Applied Mathematics, Beijing University of Technology, 100 Pingleyuan, Chaoyang District, Beijing 100124, P.R. China

e-mail: xudc@bjut.edu.cn

J. Shao

e-mail: shaojiating@bjut.edu.cn 
The facility location problem with penalties (FLPP) is introduced by Charikar et al. [3], who present a primal-dual 3-approximation algorithm. In this problem, each client can be either assigned to an open facility or reject for assignment with a penalty cost. $\mathrm{Xu}$ and $\mathrm{Xu}$ [16] further improve the approximation ratio to 1.8526. Hayrapetyan et al. [7] introduce the facility location problem with submodular penalties (FLPSP) and offer an LP-rounding 2.488-approximation algorithm. Du et al. [5] present a primal-dual combinatorial 3-approximation algorithm for the FLPSP.

In the two-stage stochastic facility location problem (SFLP), the set of clients is unknown in advance. But there are finite scenarios, each of which specified a subset of clients to be assigned. Moreover, each facility has two kinds of opening cost. One yields in the first stage and the other is scenario-dependent yielding in the second stage. The objective is to assign each client in each scenario to an open facility which is opened either in the first stage or in the second stage such that the total opening and assignment cost is minimized. Ravi and Sinha [12] give an LP-rounding 8-approximation algorithm for the SFLP.

The risk-adjusted two-stage stochastic facility location problem (RSFLP) is firstly presented by So et al. [14]. Considering that most of the recent works on the SFLP focus on minimization of the objectives of two extreme ways of handling risk, they introduce a family of risk measure functionals to interpolate between the two extreme ways and present an 8-approximation algorithm for the RSFLP.

Based on the above models, we introduce the risk-adjusted two-stage stochastic facility location problem with penalty (RSFLPP) which is an extension of the RSFLP. In the RSFLPP, a client can be assigned to an open facility (which is in the first stage or in the second stage) or rejected by paying a penalty. Integrating the LP-rounding techniques of $[13,17]$, we give a 6 -approximation algorithm for this model.

The rest of this paper is organized as followed. In Sect. 2, we describe the formulation for the RSFLPP. In Sect. 3, we give an LP-rounding-based algorithm. In Sect. 4, we analyze the algorithm and prove its approximation ratio. Some discussions are given in Sect. 5.

\section{Formulation}

In the RSFLPP, we are given a set of facilities $F$ and a set of potential clients $D$. We are also given a set of scenarios $\mathcal{S}=\{1,2, \ldots, N\}$ each of which specifies a subset of clients $D_{s} \subseteq D$ to be assigned. Each facility $i$ has an opening cost $f_{i}^{0}$ if it is opened in the first stage and $f_{i}^{s}$ if it is opened in the second stage in scenario $s$. Assigning a client $j$ to an open facility $i$ will yield an assignment cost $c_{i j}$ which satisfies the triangle inequality. Denote $p_{j}^{s}$ as the penalty incurred by the client $j \in D_{s}(s \in \mathcal{S})$ for not being assigned to any open facility. Given a constant $\alpha \in[0,1)$ which denotes the risk aversion level. We have a natural formulation for the RSFLPP as follows:

$$
\begin{array}{ll}
\min & \sum_{i \in F} f_{i}^{0} y_{i}^{0}+\beta+\frac{1}{N(1-\alpha)} \sum_{s=1}^{N} \max \left\{q\left(y^{0}, s\right)-\beta, 0\right\} \\
\text { s.t. } & y_{i}^{0} \in\{0,1\}, \quad \forall i \in F, \\
& \beta \geqslant 0,
\end{array}
$$


where

$$
\begin{array}{ll}
q\left(y^{0}, s\right):=\min \sum_{i \in F} f_{i}^{s} y_{i}^{s}+\sum_{i \in F} \sum_{j \in D_{s}} c_{i j}\left(x_{i j}^{0 s}+x_{i j}^{s s}\right)+\sum_{j \in D_{s}} p_{j}^{s} z_{j}^{s} \\
\text { s.t. } \quad \sum_{i \in F}\left(x_{i j}^{0 s}+x_{i j}^{s s}\right)+z_{j}^{s} \geqslant 1, \quad \forall j \in D_{s}, \\
\quad x_{i j}^{0 s} \leqslant y_{i}^{0}, \quad \forall i \in F, j \in D_{s}, \\
\quad x_{i j}^{s s} \leqslant y_{i}^{s}, \quad \forall i \in F, j \in D_{s}, \\
\quad x_{i j}^{0 s}, x_{i j}^{s s}, y_{i}^{s}, z_{j}^{s} \in\{0,1\}, \quad \forall i \in F, j \in D_{s} .
\end{array}
$$

Let us denote

$$
\varphi_{\alpha}\left(q\left(y^{0}, s\right)\right):=\beta+\frac{1}{N(1-\alpha)} \sum_{s=1}^{N} \max \left\{q\left(y^{0}, s\right)-\beta, 0\right\}
$$

as the risk measure function. Intuitively, $(1-\alpha)^{-1}$ is the factor which boosts the costs of scenarios in the second stage. When $\alpha=0$, the costs of scenarios in the second stage are not boosted; and as $\alpha \rightarrow 1$, the cost of scenario $s$ which has the highest cost of $q\left(y^{0}, s\right)$ is to be boosted extraordinarily. Thus $\alpha$ captures the notion of risk. $\beta$ can be viewed as the predicted cost of the second stage.

Lemma 2.1 ([14]) $\varphi_{\alpha}\left(q\left(y^{0}, s\right)\right)$ satisfies monotonicity and positive homogeneity.

(i) Monotonicity: $\varphi_{\alpha}\left(q_{1}\right) \leqslant \varphi_{\alpha}\left(q_{2}\right)$ for any random variables $q_{1}$ and $q_{2}$ with $q_{1} \leqslant$ $q_{2}$;

(ii) Positive homogeneity: $\varphi_{\alpha}(q)=t \varphi_{\alpha}(q)$ for any $t>0$ and any random variable $q$.

In formulation (2.1)-(2.2), we use $y_{i}^{0}$ (or $y_{i}^{s}$ ) to denote whether facility $i$ is open or not in the first stage (or in the second stage scenario $s$ ), $x_{i j}^{0 s}$ ( or $x_{i j}^{s s}$ ) to denote whether the client $j \in D_{s}$ is assigned to the facility $i$ or not in the first stage (or in the second stage scenario $s$ ) and $z_{j}^{s}$ to denote whether the client $j \in D_{s}$ is penalized or not. By relaxing the binary constraints, we obtain the following LP relaxation:

$$
\begin{aligned}
& \min \sum_{i \in F} f_{i}^{0} y_{i}^{0}+\beta+\frac{1}{N(1-\alpha)} \sum_{s=1}^{N} \max \left\{\sum_{i \in F} f_{i}^{s} y_{i}^{s}\right. \\
& \left.+\sum_{i \in F} \sum_{j \in D_{s}} c_{i j}\left(x_{i j}^{0 s}+x_{i j}^{s s}\right)+\sum_{j \in D_{s}} p_{j}^{s} z_{j}^{s}-\beta, 0\right\}
\end{aligned}
$$




$$
\begin{array}{ll}
\text { s.t. } & \sum_{i \in F}\left(x_{i j}^{0 s}+x_{i j}^{s s}\right)+z_{j}^{s} \geqslant 1, \quad \forall s \in \mathcal{S}, j \in D_{s}, \\
& x_{i j}^{0 s} \leqslant y_{i}^{0}, \quad \forall i \in F, s \in \mathcal{S}, j \in D_{s}, \\
& x_{i j}^{s s} \leqslant y_{i}^{s}, \quad \forall i \in F, s \in \mathcal{S}, j \in D_{s}, \\
& x_{i j}^{0 s}, x_{i j}^{s s}, y_{i}^{0}, y_{i}^{s}, z_{j}^{s}, \beta \geqslant 0, \quad \forall i \in F, s \in \mathcal{S}, j \in D_{s} .
\end{array}
$$

In the remainder of this paper, we denote $\mathcal{D}:=\left\{(j, s): s \in \mathcal{S}, j \in D_{s}\right\}$.

\section{Algorithm}

In this section, we give our algorithm for the RSFLPP which proceeds as follows. Firstly, we classify the clients set into two subsets according to the optimal solution of (2.3). The first one includes the clients to be assigned to open facilities and the second one includes those to be penalized. Secondly, we consider two cases in the first clients subset to decide which ones are to be assigned to an open facility in the first stage and which ones are to be assigned in the second stage. Finally, we get an integral feasible solution of (2.1).

\section{Algorithm 3.1}

Step 1 Given $\gamma, \eta \in(0,1)$ to be fixed later. Set $F_{0}:=\emptyset, F_{s}:=\emptyset(s \in \mathcal{S}), \mathcal{D}_{a}:=\mathcal{D}$, $\mathcal{D}_{c}^{1}:=\emptyset, \mathcal{D}_{c}^{2}:=\emptyset,(\bar{x}, \bar{y}, \bar{z}):=(0,0,0)$.

Step 2 Solve 2.3 to obtain the optimal fractional solution $(x, y, z)$. Set

$$
\begin{aligned}
\mathcal{D}_{r} & :=\left\{(j, s) \in \mathcal{D}: z_{j}^{s}>1-\gamma\right\}, \quad \mathcal{D}_{a}:=\mathcal{D}_{a} \backslash \mathcal{D}_{r}, \\
\bar{z}_{j}^{s} & :=1, \quad \forall(j, s) \in \mathcal{D}_{r} .
\end{aligned}
$$

Step 3 For each $(j, s) \in \mathcal{D}_{a}$, denote

$$
\begin{aligned}
r_{j}^{s} & :=\min _{i \in F}\left\{c_{i j}: \sum_{i^{\prime} \in F: c_{i^{\prime} j} \leqslant c_{i j}}\left(x_{i^{\prime} j}^{0 s}+x_{i^{\prime} j}^{s s}\right) \geqslant \eta \gamma\right\}, \\
N_{j}^{s 0} & :=\left\{i \in F: c_{i j} \leqslant r_{j}^{s}, x_{i j}^{0 s}>0\right\}, \\
N_{j}^{s s} & :=\left\{i \in F: c_{i j} \leqslant r_{j}^{s}, x_{i j}^{s s}>0\right\} .
\end{aligned}
$$

Step 4 Choose $(\bar{j}, \bar{s}):=\arg \min \left\{r_{j}^{s}:(j, s) \in \mathcal{D}_{a}\right\}$.

Case $1 \sum_{i \in N_{\bar{j}}^{\bar{s} 0}} x_{i}^{0 \bar{s}} \geqslant \frac{1}{2} \eta \gamma$. Choose the facility $\bar{i}:=\arg \min \left\{f_{i}^{0}: i \in N_{\bar{j}}^{\bar{s} 0}\right\}$ to open in the first stage and assign the client $(\bar{j}, \bar{s})$ to it directly. Set $\bar{x}_{\bar{i}}^{0 \bar{j}}:=1, \bar{y}_{\bar{i}}^{0}:=1$, $F_{0}:=F_{0} \cup\{\bar{i}\}, \mathcal{D}_{a}:=\mathcal{D}_{a} \backslash\{(\bar{j}, \bar{s})\}$, and $\mathcal{D}_{c}^{1}:=\mathcal{D}_{c}^{1} \cup\{(\bar{j}, \bar{s})\}$.

If there exists $(j, s) \in \mathcal{D}_{a}$ such that $N_{j}^{s 0} \cap N_{\bar{j}}^{\bar{s} 0} \neq \emptyset$, we assign the client $(j, s)$ to the facility $\bar{i}$ in the first stage indirectly. Set $\bar{x}_{\bar{i} j}^{0 s}:=1$ and $\mathcal{D}_{a}:=\mathcal{D}_{a} \backslash\{(j, s)\}$. 
Case 2 Otherwise, we have $\sum_{i \in N_{\bar{j}}^{\bar{s} s}} x_{i}^{\bar{s} \bar{j}} \geqslant \frac{1}{2} \eta \gamma$. Choose the facility $\bar{i}:=\arg \min \left\{f_{i}^{\bar{s}}\right.$ : $\left.i \in N_{\bar{j}}^{\bar{s} \bar{s}}\right\}$ to open in the second stage scenario $\bar{s}$ and assign the client $(\bar{j}, \bar{s})$ to it directly. Set $\bar{x}_{\bar{i}}^{\bar{s} \bar{j}}:=1, \bar{y}_{\bar{i}}^{\bar{s}}:=1, F_{\bar{s}}:=F_{\bar{s}} \cup\{\bar{i}\}, \mathcal{D}_{a}:=\mathcal{D}_{a} \backslash\{(\bar{j}, \bar{s})\}$, and $\mathcal{D}_{c}^{2}:=\mathcal{D}_{c}^{2} \cup\{(\bar{j}, \bar{s})\}$.

If there exists $(j, \bar{s}) \in \mathcal{D}_{a}$ such that $N_{j}^{\bar{s} \bar{s}} \cap N_{\bar{j}}^{\bar{s} \bar{s}} \neq \emptyset$, we assign the client $(j, \bar{s})$ to the facility $\bar{i}$ in the second stage indirectly. Set $\bar{x}_{\bar{i} j}^{\bar{s} \bar{s}}:=1$ and $\mathcal{D}_{a}:=\mathcal{D}_{a} \backslash\{(j, \bar{s})\}$.

Step 5 If $\mathcal{D}_{a}=\emptyset$, output $(\bar{x}, \bar{y}, \bar{z})$; otherwise, go to Step 4 .

At the end of Algorithm 3.1, we obtain a set of open facilities $F_{0}$ which open in the first stage, facilities $F_{S}$ which open in the second stage in scenario $s$, and a set of penalized clients $\mathcal{D}_{r}$.

\section{Analysis}

In this section, we give our main result addressing the approximation ratio of Algorithm 3.1. We bound the facility cost in Lemmas 4.1-4.2 and the connection cost in Lemmas 4.3-4.4. Then, by choosing suitable parameters, we draw our main theorem.

Lemma 4.1 The cost of opening facilities in the first stage is

$$
\sum_{i \in F} f_{i}^{0} \bar{y}_{i}^{0} \leqslant \frac{2}{\eta \gamma} \sum_{i \in F} f_{i}^{0} y_{i}^{0} .
$$

Proof According to Step 4 of Algorithm 3.1, if the facility $\bar{i}$ is chosen to open in the first stage, there must exist a unique client $(\bar{j}, \bar{s}) \in \mathcal{D}_{c}^{1}$ such that

$$
\sum_{i \in N_{\bar{j}}^{\bar{s} 0}} x_{i \bar{j}}^{0 \bar{s}} \geqslant \frac{1}{2} \eta \gamma
$$

If follows from Step 4 of Algorithm 3.1 and (2.3) that

$$
f_{\bar{i}}^{0} \leqslant \frac{2}{\eta \gamma} \sum_{i \in N_{\bar{j}}^{\bar{s} 0}} f_{i}^{0} x_{i}^{0 \bar{s}} \leqslant \frac{2}{\eta \gamma} \sum_{i \in N_{\bar{j}}^{\bar{s} 0}} f_{i}^{0} y_{i}^{0} .
$$

Combining the disjointness of $\left\{N_{\bar{j}}^{\bar{s} 0}\right\}_{(\bar{j}, \bar{s}) \in \mathcal{D}_{c}^{1}}$, we conclude the lemma.

Similar to Lemma 4.1, we have

Lemma 4.2 The cost of opening facilities in the second stage in scenario $s \in \mathcal{S}$ is

$$
\sum_{i \in F} f_{i}^{s} \bar{y}_{i}^{s} \leqslant \frac{2}{\eta \gamma} \sum_{i \in F} f_{i}^{s} y_{i}^{s} .
$$


Lemma 4.3 For any $(j, s) \in \mathcal{D} \backslash \mathcal{D}_{r}$ and $\bar{x}_{\bar{i} j}^{0 s}=1$, we have

$$
c_{\bar{i} j} \leqslant \frac{3}{\gamma(1-\eta)} \sum_{i \in F} c_{i j}\left(x_{i j}^{0 s}+x_{i j}^{s s}\right) .
$$

Proof We consider the following two possibilities.

Case 1 . Client $(j, s)$ is assigned to $(\bar{i}, 0)$ directly.

It follows from Step 4 of Algorithm 3.1 that $\bar{i} \in N_{j}^{s}$ which indicates that $c_{i j}^{-} \leqslant r_{j}^{s}$.

Case 2. Client $(j, s)$ is assigned to $(\bar{i}, 0)$ indirectly.

There must exist a client $\left(j^{\prime}, s^{\prime}\right) \in \mathcal{D}_{c}^{1}$ and a facility $i^{\prime}$ such that $r_{j^{\prime}}^{s^{\prime}} \leqslant r_{j}^{s}, \bar{i} \in N_{j^{\prime}}^{s^{\prime} 0}$, and $i^{\prime} \in N_{j}^{s 0} \cap N_{j^{\prime}}^{s^{\prime} 0}$. Thus we have

$$
c_{i j}^{-} \leqslant c_{i^{\prime} j}+c_{i^{\prime} j^{\prime}}+c_{\bar{i} j^{\prime}} \leqslant r_{j}^{s}+2 r_{j^{\prime}}^{s^{\prime}} \leqslant 3 r_{j}^{s} .
$$

Summing the above two cases, we have $c_{\bar{i} j} \leqslant 3 r_{j}^{s}$.

From (2.3) and the definitions of $r_{j}^{s}$ and $\mathcal{D}_{r}$, we have

$$
\sum_{i \in F: c_{i j}<r_{j}^{s}}\left(x_{i j}^{0 s}+x_{i j}^{s s}\right)<\eta \gamma
$$

and

$$
\sum_{i \in F}\left(x_{i j}^{0 s}+x_{i j}^{s s}\right)=\sum_{i \in F: c_{i j}<r_{j}^{s}}\left(x_{i j}^{0 s}+x_{i j}^{s s}\right)+\sum_{i \in F: c_{i j} \geqslant r_{j}^{s}}\left(x_{i j}^{0 s}+x_{i j}^{s s}\right) \geqslant \gamma .
$$

Thus, we have

$$
r_{j}^{s} \leqslant \frac{1}{\gamma-\eta \gamma} \sum_{i \in F: c_{i j} \geqslant r_{j}^{s}} c_{i j}\left(x_{i j}^{0 s}+x_{i j}^{s s}\right) \leqslant \frac{1}{\gamma(1-\eta)} \sum_{i \in F} c_{i j}\left(x_{i j}^{0 s}+x_{i j}^{s s}\right),
$$

which concludes the proof.

Similar to Lemma 4.3, we have

Lemma 4.4 For any $(j, s) \in \mathcal{D} \backslash \mathcal{D}_{r}$ and $\bar{x}_{\bar{i}}^{s s}=1$, we have

$$
c_{\bar{i} j} \leqslant \frac{3}{\gamma(1-\eta)} \sum_{i \in F} c_{i j}\left(x_{i j}^{0 s}+x_{i j}^{s s}\right) \text {. }
$$

Now we are ready to state our main theorem.

Theorem 4.1 Setting $\eta:=\frac{2}{5}$ and $\gamma:=\frac{5}{6}$, Algorithm 3.1 is a 6-approximation algorithm for the RSFLPP. 
Proof Noting that for any client $(j, s) \in \mathcal{D}_{r}$, we have

$$
\bar{z}_{j}^{s} \leqslant \frac{1}{1-\gamma} z_{j}^{s},
$$

which implies that

$$
\sum_{(j, s) \in \mathcal{D}} p_{j}^{s} \bar{z}_{j}^{s} \leqslant \frac{1}{1-\gamma} \sum_{(j, s) \in \mathcal{D}} p_{j}^{s} z_{j}^{s} .
$$

For any scenario $s \in \mathcal{S}$, it follows from (2.2), (4.1), and Lemmas 4.2-4.4 that

$$
\begin{aligned}
q\left(\bar{y}^{0}, s\right) & \sum_{i \in F} f_{i}^{s} \bar{y}_{i}^{s}+\sum_{i \in F} \sum_{(j, s) \in \mathcal{D}} c_{i j}\left(\bar{x}_{i j}^{0 s}+\bar{x}_{i j}^{s s}\right)+\sum_{(j, s) \in \mathcal{D}} p_{j}^{s} \bar{z}_{j}^{s} \\
\leqslant & \frac{2}{\eta \gamma} \sum_{i \in F} f_{i}^{s} y_{i}^{s}+\frac{3}{\gamma(1-\eta)} \sum_{i \in F} \sum_{(j, s) \in \mathcal{D}} c_{i j}\left(x_{i j}^{0 s}+x_{i j}^{s s}\right)+\frac{1}{1-\gamma} \sum_{(j, s) \in \mathcal{D}} p_{j}^{s} z_{j}^{s} \\
\leqslant & \max \left\{\frac{2}{\eta \gamma}, \frac{3}{\gamma(1-\eta)}, \frac{1}{1-\gamma}\right\}\left(\sum_{i \in F} f_{i}^{s} y_{i}^{s}\right. \\
& \left.+\sum_{i \in F} \sum_{(j, s) \in \mathcal{D}} c_{i j}\left(x_{i j}^{0 s}+x_{i j}^{s s}\right)+\sum_{(j, s) \in \mathcal{D}} p_{j}^{s} z_{j}^{s}\right) \\
= & 6 q\left(y^{0}, s\right) .
\end{aligned}
$$

Let $C_{\mathrm{alg}}$ and $C_{\mathrm{opt}}$ be the objective values of the integral solution $(\bar{x}, \bar{y}, \bar{z})$ of $(2.1)$ and the optimal fractional solution $(x, y, z)$ of (2.3), respectively. From (4.2), Lemmas 2.1 and 4.1, we have

$$
\begin{aligned}
C_{\mathrm{alg}} & =\sum_{i \in F} f_{i}^{0} \bar{y}_{i}^{0}+\varphi_{\alpha}\left(q\left(\bar{y}^{0}, s\right)\right) \\
& \leqslant 6 \sum_{i \in F} f_{i}^{0} y_{i}^{0}+6 \varphi_{\alpha}\left(q\left(y^{0}, s\right)\right) \\
& \leqslant 6 C_{\mathrm{opt}} .
\end{aligned}
$$

\section{Discussion}

In this paper, we present an LP-rounding 6-approximation algorithm for the RSFLPP. In the future research, it would be interesting to improve the approximation ratio for the RSFLPP. Another interesting question is to design a combinatorial approximation algorithm.

Acknowledgements The authors would like to thank the two anonymous referees for many helpful suggestions. 


\section{References}

[1] Ageev, A., Ye, Y., Zhang, J.: Improved combinatorial approximation algorithms for the $k$-level facility location problem. SIAM J. Discrete Math. 18, 207-217 (2004)

[2] Byrka, J., Aardal, K.I.: An optimal bifactor approximation algorithm for the metric uncapacitated facility location problem. SIAM J. Comput. 39, 2212-2213 (2010)

[3] Charikar, M., Khuller, S., Mount, D.M., Naraasimban, G.: Algorithms for facility location problems with outliers. In: Proc. of SODA, pp. 642-651 (2001)

[4] Chen, X., Chen, B.: Approximation algorithms for soft-capacitated facility location in capacitated network design. Algorithmica 53, 263-297 (2007)

[5] Du, D., Lu, R., Xu, D.: A primal-dual approximation algorithm for the facility location problem with submodular penalties. Algorithmica 63(1-2), 191-200 (2012)

[6] Guha, S., Khuller, S.: Greedy strike back: improved facility location algorithms. J. Algorithms 31, 228-248 (1999)

[7] Hayrapetyan, A., Swamy, C., Tardös, E.: Network design for information networks. In: Proc. of SODA, pp. 933-942 (2005)

[8] Jain, K., Mahdian, M., Markakis, E., Saberi, A., Vazirani, V.V.: Greedy facility location algorithm analyzed using dual fitting with factor-revealing LP. J. ACM 50, 795-824 (2003)

[9] Jain, K., Vazirani, V.V.: Approximation algorithms for metric facility location and $k$-median problems using the primal-dual schema and Lagrangian relaxation. J. ACM 48, 274-296 (2001)

[10] Li, S.: A 1.488-approximation algorithm for the uncapacitated facility location problem. In: Proc. of ICALP, Part II, pp. 77-88 (2011)

[11] Mahdian, M., Ye, Y., Zhang, J.: Approximation algorithms for metric facility location problems. SIAM J. Comput. 36, 411-432 (2006)

[12] Ravi, R., Sinha, A.: Hedging uncertainty: approximation algorithms for stochastic optimization programs. Math. Program. 108, 97-114 (2006)

[13] Shmoys, D.B., Tardös, E., Aardal, K.I.: Approximation algorithms for facility location problems (extended abstract). In: Proc. of STOC, pp. 265-274 (1997)

[14] So, A.M., Zhang, J., Ye, Y.: Stochastic combinatorial optimization with controllable risk aversion level. Math. Oper. Res. 34, 522-537 (2009)

[15] Sviridenko, M.: An improved approximation algorithm for the metric uncapacitated facility location problem. In: Proc. of IPCO, pp. 240-257 (2002)

[16] Xu, G., Xu, J.: An improved algorithm for uncapacitated facility location problem with penalties. Inf. Process. Lett. 94, 119-123 (2005)

[17] $\mathrm{Xu}, \mathrm{G} ., \mathrm{Xu}, \mathrm{J} .:$ An LP-rounding algorithm for approximation uncapacitated facility location problem with penalties. J. Comb. Optim. 17, 424-436 (2008) 